\title{
Context-Aware Personalized Activity Modeling in Concurrent Environment
}

\author{
Muhammad Safyan \\ Department of Computing \\ Iqra University,Islamabad, Pakistan \\ m.safyan@seecs.edu.pk \\ Sohail Sarwar \\ Department of Computing \\ Iqra University,Islamabad, Pakistan \\ sohail.sarwar@seecs.edu.pk \\ Mehtab Ahmed \\ Department of Computer Science \\ GC University,Lahore, Pakistan \\ metab.ahmed@gcu.edu.pk
}

\author{
Zia Ul Qayyum \\ Pak-UK Institute of Innovative Technologies \\ University of Gujrat, Gujrat, Pakistan \\ zia.qayyum@gmail.com
}

\author{
Muddesar Iqbal \\ School of Engineering \\ London South Bank University, UK \\ m.iqbal@isbu.ac.uk
}

\begin{abstract}
Activity recognition, having endemic impact on smart homes, faces one of the biggest challenges in learning a personalized activity model completely by using a generic model especially for parallel and interleaved activities. Furthermore, inhabitant's mistaken object interaction may entail in another spurious activity at smart homes. Identifying and removing such spurious activities is another challenging task. Knowledge driven techniques used for recognizing activity models are static in nature, lack contextual representation and may not comprehend spurious actions for parallel/interleaved activities. In this paper, a novel approach for completing the personalized model specific to each inhabitant at smart homes using generic model (incomplete) is presented that can recognize the sequential, parallel, and interleaved activities dynamically while removing the spurious activities semantically. A comprehensive set of experiments and results based upon number of correct (true positivity) or incorrect (false negativity) recognition of activities assert effectiveness of presented approach within a smart home.
\end{abstract}

Keywords- Ontology; Personalize Behaviour; Adaptive System; Concurrent Activity Recognition; Complete Activity Model

\section{INTRODUCTION}

In these days, phenomenon of "Smart-ness" is prevailing in every aspect of human life such as smart homes [1,2], smart cities [3], smart offices etc. However, our focus is "Activity Recognition" that complements a home into smart home for its inhabitants $[1,3]$.

There is couple of most prevalent approaches for activity recognition as given in the following:

A. Vision based: uses surveillance cameras to monitor the inhabitant behaviours and actions. Collection of images is used to recognise the activities by detecting regions of interest, observing the motion patterns and features like walking, hand waving and running $[5,6,7,8]$. However, these techniques have issues of privacy breaching, high bandwidth usage and cost of computing resources.
B. Sensor based: uses sensors and sensor streams [4, 9] for recognising activities. It is further divided into two categories:

a. Wearable sensors: Sensors are attached with the different part of the body for capturing their locomotion to recognise activities [10] through data mining or machine learning techniques. However, wearable sensors have issues of size, battery life, and unwillingness of inhabitant to wear them. Moreover, it is very hard to recognize the complex and similar activities with this technique such as making coffer making tea.

b. Object based sensors: Sensors are attached with home objects used by inhabitants producing certain values on interacting with those objects and sensors [11, 12]. Collection of such values is subjected to data mining techniques for activity recognition. Object based activity recognition is potentially a success due to low cost, low power, better response time and accurate measurement. Focus of our work and proposed model exploit this category of sensors for activity recognition.

Once data stream of inhabitants is received, it needs to be modelled such that a machine learning technique can learn and recognise the patterns for activity recognition. Data driven techniques [13] based on machine learning and data mining approaches. Strength these approaches is the ability to handle the noise, uncertainty, and incomplete sensor data. Others are the Knowledge driven techniques [9]: exploit rich prior knowledge in the domain of interest to construct activity models. These are semantically clear, logically elegant and easy to get started. But they are weak in handling uncertainty, temporal information and the models could be viewed as static and incomplete.

Keeping in view the issues of knowledge driven approach, a framework named "Ontology Based Concurrent Activity Recognition" (OCAR) is proposed; exploiting knowledge 
driven techniques (ontological constructs) for recognition of parallel and interleaved activities as shown in figure 1. OCAR uses generic activity model (representing minimum and necessary set of actions to be performed in every activity) by every inhabitant. Generic activity model lays the foundation for identifying personalized behaviour in conjunction with complete activity model (actions in addition with).

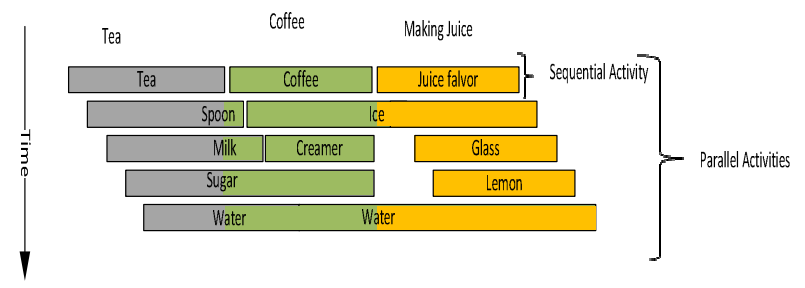

Fig 1: Parallel Activity Sensor Stream.

Concurrent and Parallel activities: move side by side with sequential activities. Inhabitants may tend to perform more than one activity simultaneously in an interleaved fashion [13] such as "making tea" activity as well as "making pasta" at the same time. All the activities have set of necessary actions (called generic model) with some additional set of inhabitant-specific actions (called personalized complete activity model). Generic model is used to devise the complete model for parallel activities.

For example, generic models for making tea $=\{$ tea, stove, cup, milk $\}$ while the behavioural model for "making tea" may refer to using inhabitant-specific objects in making tea like "white sugar" or "brown sugar" or "sweetener", "powdered milk" or "liquid milk", "strainer", "water", "spoon" etc.

One of the major approaches for AR while modelling the sensor data streams. These AR approaches are:

In addition to addressing the issue of generating a personalized complete activity model from generic model for parallel activities, OCAR addresses following research characteristics:

(1) Correct Identification of same actions belonging to different concurrent activities in a generic activity model (necessary set of actions for every activity)

(2) Distinguishing an intentional object interaction from mistaken contact by analysing the sensor stream during course of an activity e.g. Inhabitant wrongly touching the sugar jar while "making pasta" where sugar jar is used in "making tea". Such mal-interaction may be identified as sensor noise.

(3) An indispensable sensor may miss its values due to infrastructural error that might lead to incomplete activity model; system should be able to predict such incomplete action.
Rest of the paper is organized as given in the following: Section 2 provides the review of efforts made for improving AR systems. Section 3 elaborates the proposed architecture followed by implementation details in section 4 . Section 5 concludes the paper and gives future direction of work

\section{LITERATURE SURVEY}

Chen in [16] presented a hybrid approach to recognize the activities in which ontological model updates its descriptive properties based on learning the activity log file. This paper does not discuss the complete activity model of the inhabitant interim of the No. of actions used to perform the activity. Sequential activities are recognized by using the context knowledge of ontology. This paper did not discuss the parallel activities and how to handle the sensor noise.

Gorka [17] works is similar to ours in a sense, it extend the contextual knowledge through data driven techniques to recognize the personalize activity model and specialized model of an existing activity. This paper only recognize the sequential activity model and is unable to recognize the parallel activity. Author use only duration, location and activity type properties to recognize the activity but did not cater the temporal information that is integral part of activity recognition.

George[18] Combines ontological and temporal knowledge representation formalisms to provide powerful representation capabilities for composite activity modelling. Paper also presents the entailment rules to infer dynamically the composite activities. Simple activities modelled in this paper are static in nature. Our work is different from George's work in two aspects. First, we recognize complete activity model driven by generic model. Second, we recognize the simple activity in dynamic way.

Juan [19] presents a parallel AR knowledge base approach. Explore the context of the sensor activation and use context dissimilarity to cluster a continuous sensor sequence into chucks, each cluster corresponds to one under process activity. Exploit Pyramid Match Kernel approach, augmented with a WorldNet matching on hierarchical concepts, to recognize activities, using the domain ontology, from a potentially noisy sensor sequence.

In [20], the quandary of detecting concurrent activities is approached by coalescing statistical-temporal models obtained from training data and background erudition in the form of temporal first-order rules. Although the combination of data-driven and knowledge-driven solutions seems foreboding, encoding strict temporal rules often fails to integrate the level of flexibleness required in an ambient environments.

Although the combination of data- and knowledge-driven solutions seems promising, the definition of strict temporal rules often fails to incorporate the level of flexibility required in pervasive environments. 


\section{PROPOSED ARCHITECTURE}

Proposed architecture of OCAR presented in figure 2 having ability to recognize the single user sequential activities, single user concurrent activities, self-adaptive and learning for new behaviour of the inhabitant. An abstract view of operational components of proposed framework is illustrated with elaboration at finer level of granularity in the figure 2 as given below.

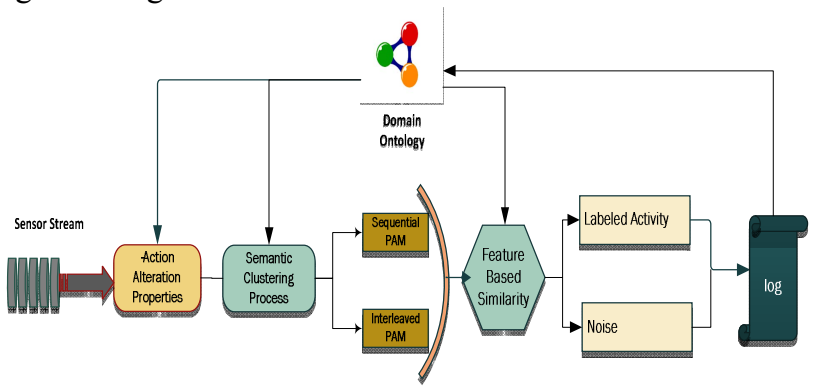

Fig 2: Proposed Architecture of Complete Activity Recognition Process

In order to provide a context to architectural components and discussion, some important definitions have been devised for implementing the concept as given in the following:

Sensor Stimulation: Sensor stimulation is triggered when a sensor changes its state from not responsive to responsive. Suppose a user takes a cup, the stimulation will be labeled as "cupSens". This sensor stimulation consists of few constraints such as timestamp $\left(\mathrm{tS}^{\circ}\right)$, sensor id (Sen-id), location $\left(\mathrm{L}^{\circ}\right)$.

$$
\begin{aligned}
& \mathrm{SS}^{\circ}=\{\text { timestamp }+ \text { sensor-id + location }\} \\
& \mathrm{SS}^{\circ}=\left\{\mathrm{tS}^{\circ}+\mathrm{Sen}-\mathrm{id}+\mathrm{L}^{\circ}\right\}
\end{aligned}
$$

Action Property: Sensor stimulation is directly linked with action properties observed as pre requisite for activities. These properties define context of object in use that helps to deduce AR process. For example, cup-sen and mug-sen are associated with action property "hasDrinkingContainer". Any given action property can be mapped with sensor stimulation through ontology context.

hasDrinkingContainer $=\{$ cup-sen, mug-sen $\}$

Perceptible Activity Model (PAM): is a set of all necessary action properties observed by the user to perform certain activity. For example, "Making Tea" activity consist of necessary action properties such as "hasDrinkingContainer", "hasHeating", "hasAdding" with sensor stimulations like "stove"," tea", "milk" and "water" whereas "sugar" is an optional ingredient. So assumption in PAM is:

PAMTea $=\{$ hasHeating(stoveSens), hasDrinkingContainer(cupSens), hasAdd(MilkSens), hasAdding(teaSens)\}

Optional Actions: are not mandatory to perform an activity but can be part of that activity. Optional actions play an integral role in recognition of a Complete Activity Model.
Complete Activity Model(CAM): Set of all necessary and optional actions performed by certain inhabitant for performing an activity. CAM may vary along each inhabitant.

CAMTea $=\{$ turnOnTap(waterSens), hasAdd(Milk), hasAdd(tea), hasDrinkingContainter(cup), hasHeating(stove), hasAdding(sugar)\}

Overlapped Actions: Set of actions in a sensor stream corresponding to two different PAMs for performing different activities in same time frame.

Activityi $=\{S$-actioni, $S$-actioni $+1 \ldots . . . S$-actionN, S-durationi $\}$ Activityj $=\{$ S-actionj, S-actionj $+1 \ldots . . S$-actionN, S-durationj $\}$

Overlapped Activity $=\{S$-actioni, S-actioni+1, S-actionj,$S$ actioni $+2, S$-actionj $+1 \ldots . S$-actioni $+N, S$-actionj $+N\}$

Erratic Behaviour/noise: sensor stimulation due to user's mistaken interaction with sensor objects that are not part of any ongoing activity.

\section{A. Ontology Activity Modeling}

Keeping above definitions in view, following ontology artefacts have been devised in the form of classes to model ontological activity model.

ADL Activities: is super concept of all simple activities performed at home like making pasta, making tea, taking bathing etc.

Object: is a super concept of entire home commodities, electrical appliance, fixtures or other day to day usable entities for performing different activities like "stove", "cup", "fridge", "waterTap".

Sensors: There are two types of sensors addressed in our model: First on/off sensors like "stove" on/off or "waterTap" on/off. Second are contact sensors producing time interval between start and end time recorded after touching certain object. Each instance of sensor class corresponds to instance of object class or its subclasses through "attachedWith" property.

Location: is super concept of different locations at home like kitchen, bathroom, TV lounge etc. Each activity must have only one location where it can be performed and each object must have only one location where it can be used.

\section{B. Action Alteration Property}

The "Action alteration Property" takes the sensor stimulation dataset and the domain knowledge ontology as input and transforms every sensor stimulation into an action property as describe in [17]. Sensor stimulation is generated as a result of user interaction with object. Each object is associated with an action property in domain ontological model. Our process transforms each sensor stimulation into corresponding property. This simple transformation is possible due to the dense sensing-based activity monitoring approach, where sensor stimulations are directly linked to user-object interactions and thus to actions. For example sensor sequence and its transformed format is: 
Sensor stream: \{cupSens, wsugarSens, smilkSens \} Transformed stream: \{hasContainer (cup), hasFlavour (white-sugar), hasMilk (skimmed-milk)\}

The result of the sensor stream transformed above remains the same even if "mugsSens", "BrownsugarSens", "LiquidMilkSens" is used instead of "cupSens", "whiteSugarSens" and "SkimmedmilkSens".

\section{Semantic Clustering Process}

This process iteratively segments the sensor stream into chunks' by using the information encoded in domain model as an ontology. For each action, its semantic compatibility is checked if action is part of any PAM; if so, actions are to be placed on one of the classes/clusters terms as a bucket labeled after PAM's name. If an action is part of multiple PAMs, multiple buckets are used to hold the bucket actions. If a bucket has all the actions of a PAM, that bucket is closed. If an action is not part of any PAM, that action is labeled as "OPTINAL".

Semantic compatibility $\left(\mathrm{S}_{\mathrm{c}}\right)$ for each actions is executed on three aspects: (i) Object compatibility $\left(\mathrm{O}_{c}\right)$ (ii) Duration compatibility $\left(\mathrm{D}_{\mathrm{c}}\right)$ and (iii) Location compatibility $\left(\mathrm{L}_{\mathrm{c}}\right)$.

$$
S_{c(0,1)}=O_{c(0,1) \wedge} D_{c(0,1) \wedge} L_{c}(0,1)
$$

Temporal logics perform better way to identify the overlapped activities as a result of erratic behaviour.

$A=$ Activity in a smart home. e.g. $A_{i}, A j$

$\mathrm{T}=$ Label of an activity A. e.g. taking medicine $=\mathrm{A}_{\mathrm{i}}$ and making $\mathrm{Tea}=\mathrm{A}_{\mathrm{j}}$

$\mathrm{a}=$ actions belong a sensor stream. E.g. $\mathrm{A}_{\mathrm{i}}=\left\{\mathrm{a}_{1}, \mathrm{a}_{2} \ldots \mathrm{an}\right\}$

$\mathrm{t}=$ time of occurrence of an action $\mathrm{A}_{\mathrm{i}}=\left\{\mathrm{a}_{1}, \mathrm{a}_{2} \ldots \mathrm{an}\right\}$ in

certain duration of an activity. E.g. $\mathrm{A}_{\mathrm{i}}=\left\{\mathrm{t}_{1}, \mathrm{t}_{2} \ldots \mathrm{tn}\right\}$

$\mathrm{D}=$ duration time to perform an Activity in context

knowledge. E.g. $\mathrm{D}_{\mathrm{i}}=\{10$ minutes $\}$

$\mathrm{d}=$ duration time (time interval) of occurrence of PAM in a sensor stream. $\mathrm{d}=\{5$ minutes $\} / /$ while Duration is 10 minutes.

$\mathrm{L}=$ location to perform an activity. E.g. making_Tea=

$\mathrm{A}_{\mathrm{j}}=\{$ kitchen $\}$.

$\mathrm{l}=$ location necessary action or optional action. $\mathrm{a}_{1}=$

$\{$ kitchen $\} \mathrm{a}_{2}=\{$ kitchen $\} \ldots$...

$p=$ is activity type i.e. property of an action/optional action in which activities it may use e.g. action "a 1 " used in \{making tea, making coffee, making pasta\}

Let assume that we have two PAM's $A_{i}$ and $A_{J}$ to describe these problems.

$\mathrm{A}_{\mathrm{i}}=\left\{\mathrm{a}_{\mathrm{i} 1}, \mathrm{a}_{\mathrm{i} 2}, \mathrm{o}_{\mathrm{i} 1} \ldots . . \mathrm{a}_{\mathrm{in}}, \ldots \mathrm{o}_{\mathrm{in}}\right\}$ at time $\left(\mathrm{t}^{\prime}{ }_{1}, \mathrm{t}^{\prime}{ }_{\left.2, \ldots . . \mathrm{t}^{\prime}{ }_{\mathrm{n} .}\right\}}\right.$

$\mathrm{A}_{\mathrm{j}}=\left\{\mathrm{a}_{\mathrm{j} 1}, \mathrm{a}_{\mathrm{j} 2}, \ldots \mathrm{O}_{\mathrm{j} 1} \ldots \mathrm{a}_{\mathrm{jn}, \ldots} \mathrm{O}_{\mathrm{jn}}\right\}$ at time $\left(\mathrm{t}^{\prime \prime}{ }_{1}, \mathrm{t}^{\prime \prime}{ }_{2}, \ldots \ldots \mathrm{t}^{\prime \prime}{ }_{\mathrm{n}}\right\}$

Two perceptible models belongs to activities $\mathrm{Ai} \& \mathrm{~A}_{\mathrm{j}}$ are partially overlaps if $\mathrm{A}_{j}$ 's necessary action starts before the completion of Ai's necessary actions. Overlapped actions may use in both activities. Figure 3 depicts the Overlapped PAM's (OVLP's)

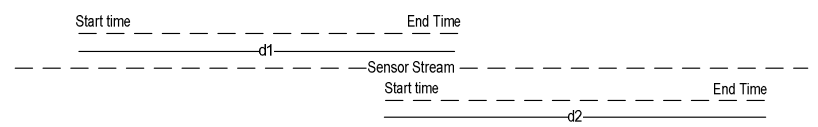

Fig.3. Overlapped PAM

Temporal formalism for the above scenario are describe below and the pseudo code to recognize this activity is

$\mathrm{A}_{\mathrm{i}}=\left\{\mathrm{a}_{\mathrm{i} 1}, \mathrm{a}_{\mathrm{i} 2}, \mathrm{a}_{\mathrm{k}} \ldots . . \mathrm{a}_{\mathrm{in}}\right\}$ at time $\left(\mathrm{t}^{\prime}{ }_{1}, \mathrm{t}^{\prime}{ }_{2} \ldots . . . \mathrm{t}^{\prime}{ }_{\mathrm{n}}\right\}$

$\mathrm{A}_{\mathrm{j}}=\left\{\mathrm{a}_{\mathrm{j} 1}, \mathrm{a}_{\mathrm{j} 2}, \mathrm{a}_{\mathrm{k}} \ldots . . \mathrm{a}_{\mathrm{j} n}\right\}$ at time $\left(\mathrm{t}^{\prime \prime}{ }_{1}, \mathrm{t}^{\prime \prime}{ }_{2} \ldots \ldots . . \mathrm{t}^{\prime \prime}{ }_{\mathrm{n}}\right\}$

$\forall\left(\mathrm{Ai}_{1} \mathrm{Aj}\right) \forall\left(\mathrm{t}^{\prime}, \mathrm{t}^{\prime \prime}\right) \quad \operatorname{Overlap}\left(\mathrm{D}_{\mathrm{i}}, \mathrm{D}_{\mathrm{j}}\right)$ partOf $\left(\mathrm{a}_{\mathrm{k}}, \mathrm{A}_{\mathrm{i}}\right) \wedge$ partOf $\left(\mathrm{a}_{\mathrm{k}}, \mathrm{A}_{\mathrm{j}}\right) \wedge\left(\right.$ during $\left.\left(\mathrm{d}_{\mathrm{i}}, \mathrm{D}_{\mathrm{i}}\right) \wedge \operatorname{during}\left(\mathrm{d}_{\mathrm{j}}, \mathrm{D}_{\mathrm{j}}\right)\right) \wedge\left(\operatorname{sameAs}\left(\mathrm{L}_{\mathrm{i}}, \mathrm{l}_{\mathrm{i}}\right) \wedge\right.$ $\left.\operatorname{same} A s\left(L_{j}, l_{j}\right)\right) \Rightarrow \operatorname{OVLP} ' s\left(A_{i}, A_{J}\right)$

\section{Feature Based Similarity}

Feature based similarity addresses the issue of overlapped PAMs that have been identified as a result of erratic behaviour

For example sensor stream " $S S$ " fulfils every criterion for two activities:

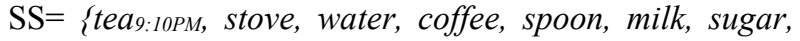
strainer, creamer, cup, ice $9: 17 P M$ \}

Stream is semantically compatible with activities of both "making coffee" and "making tea". But the time interval indicated by actions infers that only one activity is being performed. Here considerations are made on Optional Sensors (OS) for performing an activity.

Now, all the actions labeled as "OPTIONAL" within a duration space of respective activities, are assigned to their semantically compatible PAMs. If an action falls in overlapped area of two duration spaces having same semantic compatibility for both activities For example sensor stream " $S S$ "

\section{$\mathrm{SS}=\{$ tea, stove, water, coffee, spoon, milk, sugar, straniner, creamer, cup, ice\}}

Actions are semantically compatible with activities of both "making coffee" and "making tea". But the time interval indicated by actions infers that only one activity is being performed. Here considerations are made on Optional Sensors (OS) for performing an activity. We have customized Tversky's similarity [21] concepts for our activity recognition process.

$$
\operatorname{Sim} T(01,02)=\frac{\alpha(\psi(01) \cap \psi(02))}{(\beta(\psi(01) \backslash \psi(02))+\gamma(\psi(02) \backslash \psi(01))+\alpha(\psi(01) \cap \psi(02)))}
$$

Where

$\psi(\mathrm{O})$ is the function describing all the relevant features of the object $\mathrm{O}$.

$\alpha, \beta, \gamma \in \mathrm{R}$ are constants. For $\alpha=1$ common features of the two objects have maximal importance and for $\beta=\gamma$ Following notations have been used in our work: Common features of PAM and OS : $\operatorname{cf}($ PAM , OS $)=\psi$ $(\mathrm{PAM}) \cap \psi(\mathrm{OS})$, 
Distinctive features of PAM : $\mathrm{df}(\mathrm{PAM})=\psi(\mathrm{PAM}) \backslash \psi(\mathrm{OS})$ Distinctive features of OS : $\mathrm{df}(\mathrm{OS})=\psi(\mathrm{OS}) \backslash \psi(\mathrm{PAM})$. Similarity value derived from our model as given in equation (3) of Tea is 0.045 and coffee is 0.4. Tea is discarded due to less optional sensor support.

\section{RESULT AND EVALUATION}

In order to evaluate the proposed model, different aspects need to be considered such as:

- Evaluation Dataset

- Devising ground truth if a valid dataset is not available publically

- Evaluation metrics with defined inputs and outputs A. Evaluation Dataset.

To the best of our knowledge, no dataset is available publically on dataset sources like CASAS Datasets [22], Box lab or any other AR portals having noisy stream of sensor data for devising a complete activity model from generic activity model.

Therefore a hybrid evaluation methodology has been used in target environment. Survey form were used to see how the routine activities of inhabitants are performed. There has been a special focus on ensuring compliance to standards devised by KESTEREN. Salient features of our data set are given in the following:

Dataset comprised of data streams of 90 days. Parallel and sequential actions/activities were ensured through recording the streams on same day and same time interval at same and different locations.

The target activities to be evaluated are taken from kitchen such as "making tea", making coffee", "making pasta" , from bathroom such as "taking bath", "doing shave", from TV lounge such as "watching TV", "taking medicine, from bedroom such as "taking a nap" and from laundry such as "washing cloth".

\section{B. Metrics and Results}

Once data set is validated through ground truth, performance of OCAR is measured through metrics of accuracy, true positive, false positive and false negative.

The process of evaluation also covers impact of different modular units of OCAR such as semantic clustering, and feature base similarity. These units of OCAR are compared with respect to their performance on a noisy and noiseless data set, on sequential and parallel PAMs and for personalize complete activity model as well. Results on the basis of these metrics and scenarios are tabulated in table 1 .

Table 1: Complete Activity Recognition Model for Noisy and

Noiseless Parallel Scenario.

\begin{tabular}{|l|l|l|l|l|l|l|}
\hline Activities & \multicolumn{2}{|l|}{ True Positive } & \multicolumn{2}{l|}{ False Positive } & \multicolumn{2}{l|}{ False Negative } \\
\hline Making Tea & Noiseless & Noise & Noiseless & Noise & Noiseless & Noise \\
\hline Making Coffee & 96 & 95 & 1 & I & 3 & 4 \\
\hline Making Pasta & 98 & 94 & 0 & I & 5 & 5 \\
\hline
\end{tabular}

\begin{tabular}{|l|l|l|l|l|l|l|}
\hline Bathing & $97^{\prime}$ & $97^{\prime}$ & 0 & 0 & 3 & 3 \\
\hline Shaving & 98 & 98 & 0 & 0 & 2 & 2 \\
\hline Watching TV & 99 & 99 & 1 & 1 & 0 & 0 \\
\hline Take Medicine & 99 & 99 & 1 & 1 & 1 & 1 \\
\hline Washing Cloth & 99 & 99 & 0 & 0 & 1 & 1 \\
\hline Sleeping & 100 & 97 & 0 & 0 & 0 & 3 \\
\hline Chores & 100 & 98 & 0 & 0 & 0 & 2 \\
\hline
\end{tabular}

\section{Disscussion}

Now we analyse the results exhibited by OCAR as shown in tables 1 . Performance of OCAR after identifying complete activity models is presented over a mix of noisy and noisefree dataset. Performance shown by CAM is better due to dynamic duration space instead of static duration encoded in ontlogy.

\section{Conclusion}

The paper introduces a novel approach for complete activity model derived from generic activity model for parallel activities. Our proposed feature based similarity process play a significant role in eliminating those spurious activities produced as a result of sensor noise.

OCAR is presented for single user parallel activities with capacity for extending and recognizing multiple user activities in collaborative manner. Also, these activities can be extended from simple to composite ones. We look forward to complete AR process for inhabitants to extend their behavior for learning and evolving the context knowledge (modelled in ontology) by identifying the certain activities performed by certain inhabitants.

\section{REFERENCES}

[1] Gubbi, J., Buyya, R., Marusic, S. and Palaniswami, M., 2013. Internet of Things (IoT): A vision, architectural elements, and future directions. Future generation computer systems, 29(7), pp.1645-1660.

[2] Chen, Liming, Chris D. Nugent, and Hui Wang. "A knowledgedriven approach to activity recognition in smart homes." IEEE Transactions on Knowledge and Data Engineering 24.6 (2012): 961974.

[3] Caragliu, A., Del Bo, C. and Nijkamp, P., 2011. Smart cities in Europe. Journal of urban technology, 18(2), pp.65-82.

[4] [4] Gu, T., Wang, L., Wu, Z., Tao, X., Lu, J. (2011) A Pattern Mining Approach to Sensor-Based Human Activity Recognition. IEEE Transactions on Knowledge and Data Engineering, 23(9), 13591372 .

[5] B. Georis, M. Maziere, F. Bremond, and M. Thonnat, "A video interpretation platform applied to bank agency monitoring," in Proc. 2nd Workshop Intell. Distributed Surveillance Syst., 2004, pp. 46-50.

[6] J. Hobbs, R. Nevatia, and B. Bolles, "An ontology for video event representation,” in Proc. IEEE Workshop Event Detection Recognit., 2004,p. 119.

[7] A. R. J. Francois, R. Nevatia, J. Hobbs, and R. C. Bolles, "Verl: An ontology framework for representing and annotating video events," IEEE MultiMedia Mag., vol. 12, no. 4, pp. 76-86, Oct.-Dec. 2005.

[8] Poppe, R., 2010. A survey on vision-based human action recognition. Image and vision Pantelopoulos, A. and Bourbakis, N.G., 2010. 
[9] Chen, L., Hoey, J., Nugent, C.D., Cook, D.J. and Yu, Z., 2012. Sensor-based activity recognition. IEEE Transactions on Systems, Man, and Cybernetics, Part C (Applications and Reviews), 42(6), pp.790-808.

[10] Pantelopoulos, A. and Bourbakis, N.G., 2010. A survey on wearable sensor-based systems for health monitoring and prognosis. IEEE Transactions on Systems, Man, and Cybernetics, Part C (Applications and Reviews), 40(1), pp.1-12.

[11] Riboni, Daniele, et al. "Is ontology-based activity recognition really ffective?." Pervasive Computing and Communications Workshops (PERCOM Workshops), IEEE International Conference on. IEEE, 2011.

[12] Chen, L. and Nugent, C., 2009. Ontology-based activity recognition in intelligent pervasive environments. International Journal of Web Information Systems, 5(4), pp.410-430.

[13] Gu, T., Wu, Z., Tao, X., Pung, H.K. and Lu, J., 2009, March. epsicar: An emerging patterns based approach to sequential, interleaved and concurrent activity recognition. In Pervasive Computing and Communications, 2009. PerCom 2009. IEEE International Conference on (pp. 1-9). IEEE.

[14] Bao, L. and Intille, S.S., 2004, April. Activity recognition from userannotated acceleration data. In International Conference on Pervasive Computing (pp. 1-17). Springer Berlin Heidelberg.

[15] Khan, S. and Safyan, M., 2014. Semantic matching in hierarchical ontologies. Journal of King Saud University-Computer and Information Sciences, 26(3), pp.247-257.
[16] Chen, L., Nugent, C. and Okeyo, G., 2014. An ontology-based hybrid approach to activity modeling for smart homes. IEEE Transactions on human-machine systems, 44(1), pp.92-105.

[17] Azkune, Gorka, et al. "Extending knowledge-driven activity models through data-driven learning techniques." Expert Systems with Applications 42.6 (2015): 3115-3128.

[18] Okeyo, G., Chen, L., Wang, H., Sterritt, R. (2012) A KnowledgeDriven Approach to Composite Activity Recognition in Smart Environments. Ubiquitous Computing and Ambient Intelligence. Vol. 7656 of the series Lecture Notes in Computer Science. Springer Berlin Heidelberg. pp. 322-329.

[19] Ye, Juan, Graeme Stevenson, and Simon Dobson. "KCAR: A knowledge-driven approach for concurrent activity recognition." Pervasive and Mobile Computing 19 (2015): 47-70.

[20] Meditskos, G., Kontopoulos, E. and Kompatsiaris, , 2015. ReDef: Context-aware Recognition of Interleaved Activities using OWL 2 and Defeasible Reasoning. In SSN-TC/OrdRing@ ISWC (pp. 31-42).

[21] Likavec, Silvia, and Federica Cena. "Property-based Semantic Similarity: What Counts?." AIC. 2015.

[22] Online referencing http://ailab.wsu.edu/casas/datasets/ (accessed February 2017) 